\title{
Gastric relaxation in response to chemical stimulation of the area postrema in the rabbit
}

\author{
Fulvia Bongianni a, Donatella Mutolo a , Meera Srinivasan ${ }^{\mathrm{b}}$, Gabriele Staderini a, \\ Maria C. Baccari ${ }^{a}$, Franco Calamai ${ }^{a}$, Tito Pantaleo ${ }^{a, *}$ \\ a Dipartimento di Scienze Fisiologiche, Università di Firenze, Viale G.B. Morgagni 63, I-50134 Florence, Italy, \\ ${ }^{b}$ Nobel Institute for Neurophysiology, Karolinska Institutet, S-104 01, Stockholm, Sweden
}

(Accepted 22 February 1994)

\begin{abstract}
Microinjections of DL-homocysteic acid into the area postrema (AP) of anesthetized rabbits provoked gastric relaxations associated with small changes in blood pressure and marked excitatory effects on respiration. Both gastric and cardiovascular effects failed to occur after bilateral vagotomy. Comparable gastric relaxations were induced before and after treatment with atropine or atropine and guanethidine. The AP appears to play a role in gastric motility via vagus nerves and nonadrenergic noncholinergic intramural inhibitory neurons.
\end{abstract}

Key words: Area postrema; Gastric relaxation; Control of gastric motility; Circumventricular organ; Vagus nerve; DL-Homocysteic acid

The area postrema (AP) is the most caudal of the circumventricular organs and lies outside the bloodbrain barrier [22]. For several decades, the primary function attributed to the AP has been that of a chemoceptive trigger zone for emesis [8]. Subsequently, this region has been implicated in various other physiological functions [7], such as cardiovascular regulation $[5,10,11,21]$. More recently, we have provided evidence for its role in the control of respiration [20].

The influence of the AP on gastric motility has not yet been investigated directly: its role in the control of gastric relaxation has been inferred from vagally mediated changes in gastric motility accompanying vomiting or under other circumstances [1,2].

The present study was undertaken to investigate the role of the AP in the control of gastric motility in the rabbit. For this purpose we have made use of microinjections of the broad-spectrum excitatory amino acid agonist, DL-homocysteic acid (DLH) which excites cell bodies and not axons of passage $[13,23]$.

\footnotetext{
* Corresponding author. Fax: (39) (55) 437-9506.
}

Experiments were carried out on sixteen male New Zealand white rabbits $(2.5-3.4 \mathrm{~kg})$ anesthetized with a mixture of $\alpha$-chloralose ( $40 \mathrm{mg} / \mathrm{kg}$; Sigma) and urethane $(800 \mathrm{mg} / \mathrm{kg}$; Sigma) given intravenously and supplemented when necessary $(4 \mathrm{mg} / \mathrm{kg}$ and $80 \mathrm{mg} / \mathrm{kg}$, respectively). The state of the pupil (constriction) and the absence of the reflex withdrawal of the hindlimbs to nociceptive stimuli ensured full surgical anesthesia. Following cannulation of the trachea, polyethylene catheters were inserted into a femoral artery and vein for recording of arterial blood pressure and systemic administration of drugs, respectively. Both $\mathrm{C}_{5}$ phrenic roots were dissected free, cut distally and prepared for recordings. Both cervical vagus nerves were separated from the sympathetic trunks for subsequent vagotomy. The animal was placed in a prone position and fixed by a stereotaxic head-holder and vertebral clamps. The medulla was widely exposed by occipital craniotomy. Body temperature was maintained at $38 \pm 0.5^{\circ} \mathrm{C}$ by a heating blanket controlled through a rectal thermistor probe. During the course of most experiments the vagi were ligated and cut centrally; the vagotomized animals were paralysed with gallamine triethiodide (initial 
dose $4 \mathrm{mg} / \mathrm{kg}$ i.v., supplemented with $2 \mathrm{mg} / \mathrm{kg}$ every $30 \mathrm{~min}$; Sigma) and artificially ventilated. In paralysed animals, the depth of anesthesia was continuously assessed by monitoring a stable and regular pattern of phrenic discharge as well as the absence of fluctuations in arterial blood pressure whether spontaneous or in response to somatic nociceptive stimuli. To block gastric responses mediated by cholinergic and adrenergic nerves [2] we administered intravenously (slow infusion, over 1-2 $\mathrm{min}$ ) atropine sulphate ( $2 \mathrm{mg} / \mathrm{kg}$; Sigma $)$ and guanethidine sulphate $(0.8 \mathrm{mg} / \mathrm{kg}$; Ciba $)$, respectively; these doses were chosen on the basis of previous observations in the rabbit $[4,6]$. The administration of these drugs was repeated during the course of the experiment when necessary.

Efferent phrenic nerve activity was recorded from desheathed $\mathrm{C}_{5}$ phrenic roots using bipolar platinum electrodes, amplified, full-wave rectified and integrated (low-pass filter, time constant $100 \mathrm{~ms}$ ). Arterial blood pressure was recorded by a strain gauge manometer. End-tidal $\mathrm{CO}_{2}$ was monitored by means of an infrared $\mathrm{CO}_{2}$ analyzer. Intragastric pressure was recorded using a large flaccid (rubber) balloon passed down the oesophagus into the stomach and connected via non-distensible plastic tubing to a strain gauge manometer ('isometric' recording). The balloon was filled with $50-70 \mathrm{ml}$ of warm water $\left(38^{\circ} \mathrm{C}\right)$; the volume was adjusted to give an intragastric resting pressure of $5-8 \mathrm{~cm}$ $\mathrm{H}_{2} \mathrm{O}$. The signals of all variables studied were continuously monitored on a 8-channel rectilinearly writing chart recorder.

Stimulation procedures have been fully described in a previous report [20]. Microinjections (5-20 nl) of DL-homocysteic acid (DLH; Sigma) $160 \mathrm{mM}[13,16]$ were performed into the AP at appropriate depths $(150-300 \mu \mathrm{m})$. The drug was dissolved in $0.9 \% \mathrm{NaCl}$ solution or in mock cerebrospinal fluid [17] containing in most experiments $0.1-0.2 \%$ Pontamine sky blue to mark the injection site. The $\mathrm{pH}$ was adjusted to 7.4 with $1 \mathrm{~N} \mathrm{NaOH}$. The injections were made under dissecting microscope control $(\times 40)$ to evaluate the presence and spread of the injectate, via a glass micropipette (tip diameter $10-25 \mu \mathrm{m}$ ) by applying pressure, using an air-filled syringe connected by polyethylene tubing. The time taken to inject the solution ranged from 5 to $10 \mathrm{~s}$. The volume of the injectate was measured directly by monitoring the movement of the fluid meniscus in the pipette barrel with a microscope equipped with a fine reticle. Control injections of equal volumes of vehicle solutions were also performed.

At the end of the experiment the brain was removed and fixed in $10 \%$ formol saline. Frozen coronal sections (20 $\mu \mathrm{m}$ thick) stained with Cresyl violet were used for the histological control of pipette tracks and injection sites. The atlas of Shek et al. [19] was used for comparison.
Since we had already reported on respiratory responses induced by chemical stimulation of the AP [20], in the present study our analysis was focused mainly on changes in intragastric pressure and, to a minor extent, in arterial blood pressure. Systolic blood pressure and intragastric pressure were measured, at 2 $\mathrm{s}$ intervals, for $20 \mathrm{~s}$ and averaged both in the period immediately preceding each trial and during chemical stimulation at the time when the maximum response occurred (see below). Owing to the small variations in both studied variables within a measurement period, the average value for each period was treated as a single measurement for purpose of analysis. All data are expressed as means \pm S.E.M. Comparisons were made by means of Wilcoxon signed rank tests or Mann-Whitney tests; $P<0.05$ was considered as significant.

Microinjections $(n=16)$ of $160 \mathrm{mM} \mathrm{DLH} \mathrm{(5-20} \mathrm{nl,}$ 0.8-3.2 nmol) into the AP (mid-line or just lateral to the mid-line, $>0.6 \mathrm{~mm}$ caudal to the rostral margin of the AP at 150-300 $\mu \mathrm{m}$ depth) of eleven anesthetized and spontaneously breathing rabbits induced a complex pattern of response (Fig. 1A). The fully developed Aresponse was characterized by an obvious $(P<0.001)$ gastric relaxation $\left(2.8 \pm 0.2 \mathrm{~cm} \mathrm{H}_{2} \mathrm{O}\right.$, range $2-3.8 \mathrm{~cm}$ $\mathrm{H}_{2} \mathrm{O}$ ) associated with marked excitatory effects on phrenic nerve activity and small but significant $(P<$ $0.01)$ increases in arterial blood pressure $(8 \pm 1.5$ $\mathrm{mmHg}$, range $5-15 \mathrm{mmHg}$ ). The respiratory effects were similar to those previously obtained in vagotomized and paralysed rabbits [20]; they consisted of progressive and marked increases in respiratory frequency, usually associated with the development of tonic phrenic activity (Fig. 1A). The onset of gastric relaxation was relatively sharp and rapid with an average latency (from the start of the injection) of $12.2 \pm 0.5$ $\mathrm{s}$ (range $10-15 \mathrm{~s})$. It accompanied $(n=4)$ or more often $(n=12)$ preceded that of respiratory effects by a few to $25 \mathrm{~s}$. The increase in arterial blood pressure developed more gradually with an onset latency difficult to define. In the period immediately following the injection, changes in arterial blood pressure were short-lasting and inconsistent: arterial pressure was either unaffected $(n=7)$ or slightly $(\leq 5 \mathrm{mmHg})$ decreased $(n=9)$. The maximum response in gastric motility occurred within $30-110 \mathrm{~s}$ (average $62.4 \pm 4.8 \mathrm{~s}$ ) from the start of the injection; at that time also cardiorespiratory effects had attained their maximum level. All studied variables displayed a similar gradual recovery which was completed within $15-20 \mathrm{~min}$. Only microinjections generating a clear blue spot of dye confined within the parenchyma of the AP (as observed under the dissecting microscope control) were consistently associated with this pattern of response. The location of the injection sites was confirmed by the histological analysis. These responses to DLH microin- jectio anima the sa sufficis Contro locatio

Mic but in the lat to it, e tric rel contrac induce increas phasic Howev case a

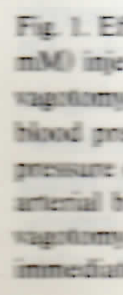


jections were reproducible both within and between animals; the same response to repeated injections at the same location was obtained, provided an interval sufficient to achieve complete recovery was allowed. Control microinjections of vehicle solutions at the same locations did not induce any appreciable effect.

Microinjections $(n=21)$ of DLH at similar depths but in close neighbouring regions, along tracks through the lateral border of the AP and up to $0.5 \mathrm{~mm}$ lateral to it, elicited varied responses in gastric motility. Gastric relaxation $(n=6)$ or biphasic responses $(n=9)$, i.e. contraction followed by relaxation or vice versa were induced at more medial injection sites, while sustained increases in gastric tone sometimes with superimposed phasic contractions $(n=6)$ at more lateral locations. However, all these gastric motor responses were in no case accompanied by excitatory effects on respiration; on the contrary, as expected from our previous findings [20], phrenic nerve activity always showed marked depressant responses characterized mainly by a decrease in frequency. In addition, changes in gastric motility occurred in most cases $(n=11)$ without concomitant changes in blood pressure and more seldom in association with marked $(\geq 30 \mathrm{mmHg}$ ) pressor responses $(n=$ $4)$ or even with slight decreases in blood pressure $(n=6)$ at sites more lateral to the AP.

Most of the animals ( $n=8)$ were also paralysed and artificially ventilated during the course of the experiment. Chemical stimulation of the AP induced similar gastric relaxations $\left(2.6 \pm 0.3 \mathrm{~cm} \mathrm{H}_{2} \mathrm{O}\right)$ in these preparations; changes in arterial blood pressure displayed similar features, but were less obvious $(\leq 5 \mathrm{mmHg})$ and did not reach statistical significance. DLH microinjections into the AP performed at the same site after
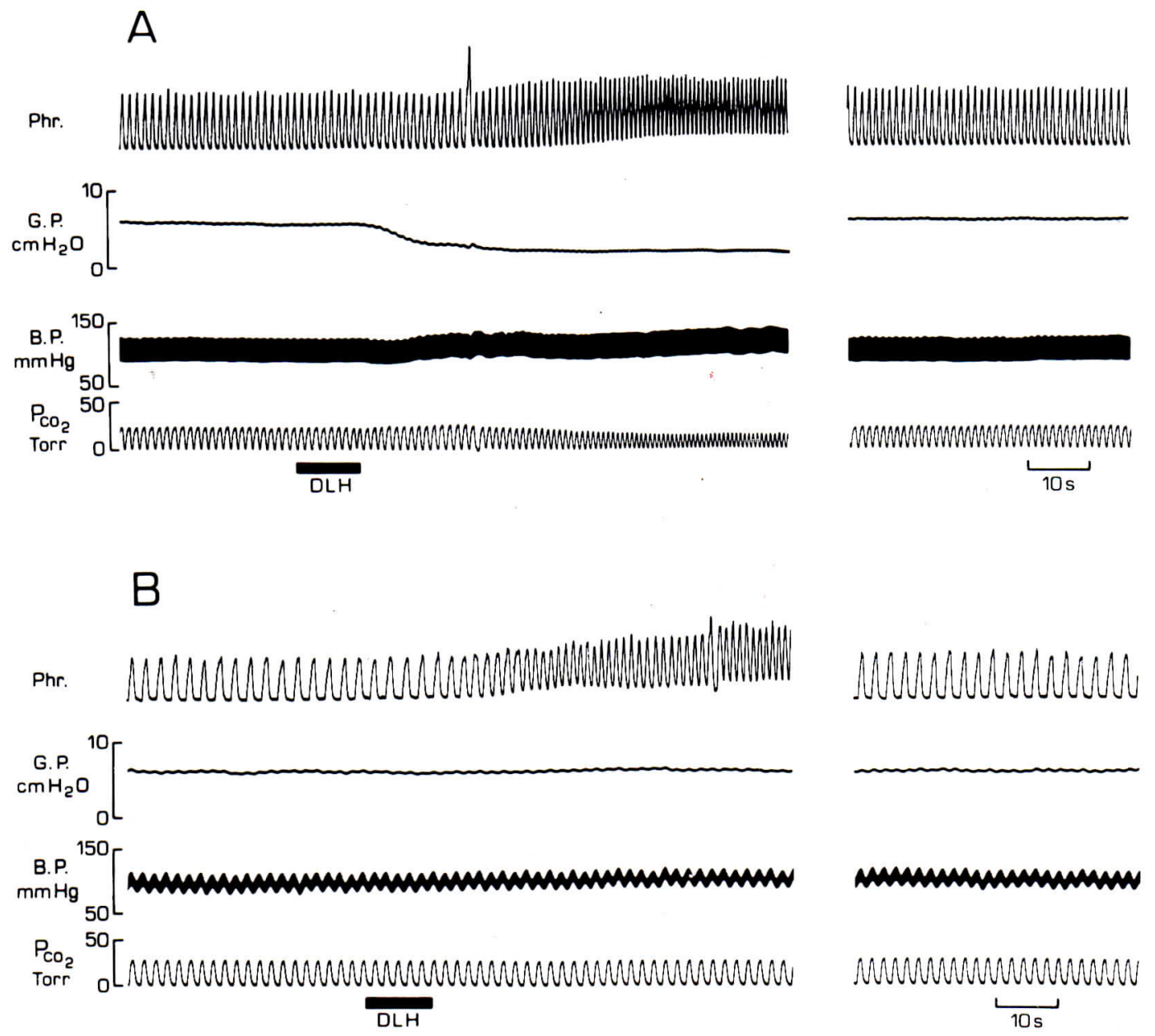

Fig. 1. Effects on gastric pressure (G.P.), integrated phrenic nerve activity (Phr.) and arterial blood pressure (B.P.) in response to $10 \mathrm{nl}$ DLH (160 $\mathrm{mM})$ injected into the AP $(1.0 \mathrm{~mm}$ caudal to the rostral border of AP, on the mid-line at $200 \mu \mathrm{m}$ depth $)$ in the same rabbit before and after vagotomy. A: decrease in gastric pressure associated with excitatory effects on integrated phrenic nerve activity and small increase in arterial blood pressure. Anesthetized, spontaneously breathing preparation. The increase in ventilation induced obvious decreases in end-tidal partial pressure of carbon dioxide $\left(\mathrm{p}_{\mathrm{CO} 2}\right)$. B: excitatory effects on integrated phrenic nerve activity, without concomitant changes in gastric pressure and arterial blood pressure. Anesthetized, bilaterally vagotomized and paralysed animal (gain of integrated phrenic nerve activity readjusted after vagotomy). The absence of changes in end-tidal $\mathrm{p}_{\mathrm{CO} 2}$ is related to the artificial ventilation of the animal. Left panels, control and effects immediately following the injection (filled bar). Right panels, recovery $16 \mathrm{~min}$ after completion of the injection. 
bilateral vagotomy failed to induce both gastric relaxation and any change in arterial blood pressure. On the other hand, evident excitatory responses in respiratory activity were elicited (Fig. 1B), in agreement with our previous findings [20]. Representative injection sites at which DLH microinjections elicited changes in gastric motility are diagrammatically illustrated in Fig. 2.

Similar gastric relaxations and changes in blood pressure in response to chemical stimulation of the AP were observed in a separate group of anesthetized, paralysed and artificially ventilated animals $(n=5)$. In these preparations, comparable gastric relaxations were produced by AP chemical stimulation at the same location before $\left(2.6 \pm 0.3 \mathrm{~cm} \mathrm{H}_{2} \mathrm{O}\right)$ and after treatment with either atropine alone $\left(2.4 \pm 0.3 \mathrm{~cm} \mathrm{H}_{2} \mathrm{O}\right)$ or atropine and guanethidine $\left(2.5 \pm 0.2 \mathrm{~cm} \mathrm{H}_{2} \mathrm{O}\right)$. No ap-
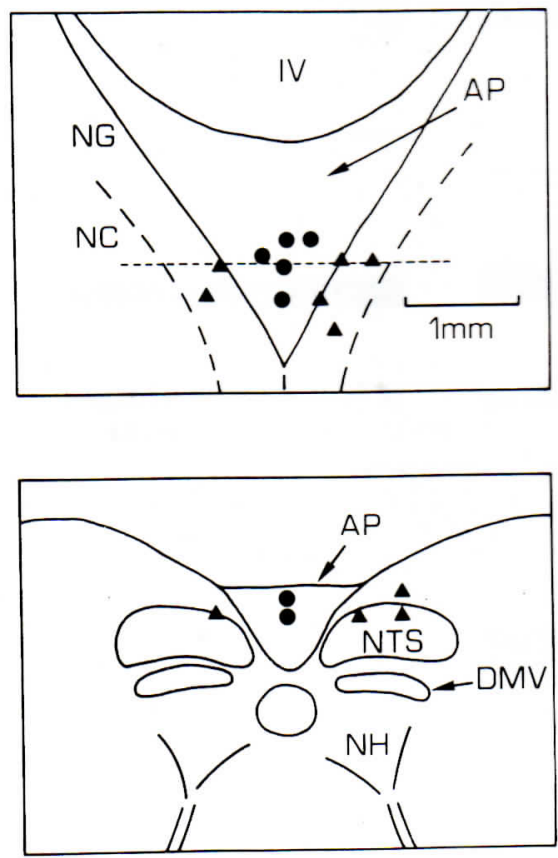

Fig. 2. Diagrammatic illustration of representative injection sites projected on a dorsal view of the AP (upper panel) and on a frontal section of the medulla at about $1.1 \mathrm{~mm}$ caudal to the rostral border of the AP (lower panel). The outlines of the maps derive from the atlas of Shek et al. [19]. In the lower panel representative sites of injections performed at various depths in close vicinity of the selected frontal section (horizontal dashed line in the upper panel) have been reported; it is shown that injections performed along tracks close to or through the lateral border of the AP affected close neighbouring areas, such as the medial aspect of the nuclear complex of the solitary tract. $\bullet$, gastric relaxation associated with excitatory effects on respiratory activity; $\boldsymbol{\Delta}$, various types of change in gastric motility associated with depressant effects on respiratory activity. $\mathrm{AP}$, area postrema; DMV, dorsal motor nucleus of the vagus nerve; $\mathrm{NC}$, nucleus cuneatus; NG, nucleus gracilis; $\mathrm{NH}$, nucleus nervi hypoglossi; NTS, nucleus tractus solitarii; IV, fourth ventricle. preciable changes in arterial blood pressure were evoked under either treatment. Drug infusion per se did not significantly alter basal gastric pressure; atropine produced either no change or small and transient (3-4 $\mathrm{min}$ ) decreases in gastric pressure, while guanethidine induced small, varying and inconsistent changes. In any case, at the time of the AP chemical stimulation resting gastric pressure had recovered control values, thus not contributing to affect DLH-induced gastric relaxations [3].

These results in conjunction with the histological verification of the injection sites make us confident that the effects obtained in response to chemical stimulation of the AP are specific and are related to the activation of the AP neurons. We have recently reported [20] a complete discussion on the methodology employed in chemical stimulation of the AP. The main concern is that pressure injections of excitatory amino acids can produce depolarization block of neurons in the immediate vicinity of the pipette tip [16]. This implies that the response may result from an inhibition rather than an activation of neurons. In the present study, we have always injected $<4 \mathrm{nmol}$ of DLH in volumes $\leq 20 \mathrm{nl}$ to minimize the risk of depolarization block generation [16] and to restrict the spread of the injectate.

Present data confirm that the AP plays a role in cardiovascular $[5,7,10,11,21]$ and respiratory [20] control. The early slight decreases observed in arterial blood pressure, although statistically non-significant, are consistent with some previous reports on cardiovascular changes induced by AP electrical or chemical stimulation $[10,14,21]$. Our results in paralysed animals and in paralysed and vagotomized preparations suggest that the small DLH-induced pressor responses obtained in this study are, to a great extent, secondary to mechanical changes due to increased respiratory activity, but they do not completely rule out a role of vagus nerves in their mediation. However, it remains to be elucidated why varied cardiovascular responses have been described in different animal species $[5,10,11,14$, 21].

The main conclusion of the present study is that DLH-induced activation of cell bodies within the AP elicits a rapid and prolonged gastric relaxation closely associated with characteristic respiratory effects. We provide here the first direct evidence that the AP influences gastric motility. In addition, we show that this influence is vagally mediated and implies a role of the vagal drive to the nonadrenergic noncholinergic intramural inhibitory neurons $[2,4]$. This role of the AP in the control of gastric motility is not surprising in view of its well-established emetic function [7,8]. However, it seems appropriate that this function is being exhibited also in species that do not have an emetic reflex (e.g. the rabbit and the rat) [9] under other

circum toxic :

Th

sopha conne syste tarii of ga vagu nucl

and AP well cent stim

ter

nol

$\mathrm{M}$.

(co

of

[1 
circumstances [2] such as, for instance, the response to toxic agents or to food intake.

The AP receives significant visceral (vagal, glossopharyngeal) afferent projections and has extensive connections with other regions of the central nervous system $[9,12,15,18]$, including the nucleus tractus solitarii and other neural structures involved in the control of gastric motility, e.g. the dorsal motor nucleus of the vagus nerve, the nucleus ambiguus and the retrofacial nucleus $[9,12]$. Thus, owing to its neuronal connections and location outside the blood-brain barrier [22], the AP could have a role in triggering gastric relaxation, as well as other autonomic functions, in response to both central or peripheral neural inputs and blood-borne stimuli.

This study was supported by grants from the Ministero della Università e della Ricerca Scientifica e Tec- * nologica of Italy and Svenska Läkaresällskapet, 868.0. M.S. was the recipient of a Swedish-Italian Fellowship (cooperation between the National Research Council of Italy and Medical Research Council of Sweden).

[1] Abrahamsson, H., Jansson, G. and Martinson, J., Vagal relaxation of the stomach induced by apomorphine in the cat, Acta Physiol. Scand., 88 (1973) 296-302.

[2] Andrews, P.L.R., Central organization of the vagal drive to the nonadrenergic noncholinergic neurones controlling gastric motility, Arch. Int. Pharmacodyn., 303 (1990) 167-198.

[3] Andrews, P.L.R. and Lawes, I.N.C., Gastric tone modifies the responses to extrinsic neural stimuli in the anaesthetized ferret, J. Physiol., 366 (1985) 1-16.

[4] Baccari, M.C., Calamai, F. and Staderini, G., The influence of the vagally induced rebound contractions on the non-adrenergic, non-cholinergic (NANC) inhibitory motility of the rabbit stomach and the role of prostaglandins, J. Auton. Nerv. Syst., 37 (1992) 125-136.

[5] Barnes, K.L., Ferrario, C.M. and Conomy, J.P., Comparison of the hemodynamic changes produced by electrical stimulation of the area postrema and nucleus tractus solitarii in the dog, Circ. Res., 45 (1979) 136-143.

[6] Beck, K., Calamai, F., Staderini, G. and Susini, T., Gastric motor responses elicited by vagal stimulation and purine compounds in the atropine-treated rabbit, Br. J. Pharmacol. 94 (1988) 1157-1166.

[7] Borison, H.L., Area postrema: chemoreceptor circumventricular organ of the medulla oblongata, Prog. Neurobiol., 32 (1989) $351-390$.
[8] Borison, H.L. and Wang, S.C., Physiology and pharmacology of vomiting, Pharmacol. Rev., 5 (1953) 193-230.

[9] Carpenter, D.O., Central nervous system mechanisms in deglutition and emesis. In S.G. Schultz (Ed.), Handbook of Physiology, Section 6, The Gastrointestinal System, Vol. I, Motility and Circulation, Am. Physiol. Soc., Bethesda, 1989, pp. 685-714.

[10] Ferguson, A.V. and Marcus, P., Area postrema stimulation induced cardiovascular changes in the rat, Am. J. Physiol., 255 (Integrative Comp. Physiol. 24) (1988) R855-R860.

[11] Gatti, P.J., Souza, J.D., da Silva, A.M.T., Quest, J.A. and Gillis, R.A., Chemical stimulation of the area postrema induces cardiorespiratory changes in the cat, Brain Res., 346 (1985) 115-123.

[12] Gillis, R.A., Quest, J.A., Pagani, F.D. and Norman, W.P., Control centers in the central nervous system for regulating gastrointestinal motility. In S.G. Schultz (Ed.), Handbook of Physiology, Section 6, The Gastrointestinal System, Vol. I, Motility and Circulation, American Physiological Society, Bethesda, 1989, pp. 621-684.

[13] Goodchild, A.K., Dampney, R.A.L. and Bandler, R.A., A method for evoking physiological responses by stimulation of cell bodies, but not axons of passage within localized regions of the central nervous system, J. Neurosci. Methods, 6 (1982) 351363.

[14] Hasser, E.M., Nelson, D.O., Haywood, J.R. and Bishop, V.S., Inhibition of renal sympathetic nervous activity by area postrema stimulation in rabbits, Am. J. Physiol., 253 (Integrative Comp. Physiol. 22) (1987) H91-H99.

[15] Leslie, R.A. and Gwyn, D.G., Neuronal connections of the area postrema, Fed. Proc., 43 (1984) 2941-2943.

[16] Lipski, J., Bellingham, M.C., West, M.J. and Pilowsky, P., Limitations of the technique of pressure microinjection of excitatory amino acids for evoking responses from localized regions of the central nervous system, J. Neurosci. Methods, 26 (1988) 169-179.

[17] Mitchell, R.A., Loeschcke, H.H., Massion, W.H. and Severinghaus, J.W., Respiratory responses mediated through superficial chemosensitive areas on the medulla, J. Appl. Physiol., 18 (1963) 523-533.

[18] Morest, D.K., Experimental study of the projections of the nucleus tractus solitarius and the area postrema in the cat, $J$. Comp. Neurol., 130 (1967) 277-299.

[19] Shek, J.W., Wen, G.Y. and Wisniewski, H.M., Atlas of the Rabbit Brain and Spinal Cord, Karger, Basel, 1986.

[20] Srinivasan, M., Bongianni, F., Fontana, G.A. and Pantaleo, T., Respiratory responses to electrical and chemical stimulation of the area postrema in the rabbit, J. Physiol., 463 (1993) 409-420.

[21] Sun, M.K. and Spyer, K.M., GABA-mediated inhibition of medullary vasomotor neurones by area postrema stimulation in rats, J. Physiol., 436 (1991) 669-684.

[22] Wislocki, G.B. and Putnam, T.J., Note on the anatomy of the areae postremae, Anatom. Rec., 19 (1920) 281-287.

[23] Zieglgansberger, W. and Puil, E.A., Actions of glutamic acid on spinal neurones, Exp. Brain Res., 17 (1973) 35-49. 